Algebraic $8 \mathcal{G}$ Geometric $\mathcal{T}_{\text {opology }}$

Volume 5 (2005) 355-368

Published: 21 April 2005

ATG

\title{
Geography of symplectic 4-manifolds with Kodaira dimension one
}

\author{
ScotT BALDRIDGE \\ TIAN-JUN LI
}

\begin{abstract}
The geography problem is usually stated for simply connected symplectic 4-manifolds. When the first cohomology is nontrivial, however, one can restate the problem taking into account how close the symplectic manifold is to satisfying the conclusion of the Hard Lefschetz Theorem, which is measured by a nonnegative integer called the degeneracy. In this paper we include the degeneracy as an extra parameter in the geography problem and show how to fill out the geography of symplectic 4-manifolds with Kodaira dimension 1 for all admissible triples.
\end{abstract}

AMS Classification 57R17; 53D05, 57R57, 57M60

Keywords Symplectic 4-manifolds, symplectic topology

\section{Introduction}

For a minimal symplectic 4-manifold $M$ with symplectic form $\omega$ and symplectic canonical class $K_{\omega}$, the Kodaira dimension of $(M, \omega)$ is defined in the following way:

$$
\kappa(M, \omega)=\left\{\begin{array}{cllll}
-\infty & \text { if } & K_{\omega}^{2}<0 & \text { or } & K_{\omega} \cdot[\omega]<0 \\
0 & \text { if } & K_{\omega}^{2}=0 & \text { and } & K_{\omega} \cdot[\omega]=0 \\
1 & \text { if } & K_{\omega}^{2}=0 & \text { and } & K_{\omega} \cdot[\omega]>0 \\
2 & \text { if } & K_{\omega}^{2}>0 & \text { and } & K_{\omega} \cdot[\omega]>0
\end{array}\right.
$$

The Kodaira dimension of a non-minimal manifold is defined to be that of any of its minimal models (see [12, [8]).

Minimal symplectic manifolds of Kodaira dimension $-\infty$ were classified in [10]. Such manifolds are either $\mathbf{C P}^{2}$ or an $S^{2}$-bundle over a surface. Minimal symplectic manifolds of Kodaira dimension zero were studied in [8]: it was speculated that they are either K3, Enriques surface or a $T^{2}$-bundle over $T^{2}$; and it was shown that the $\chi$ and $\sigma$ are bounded if $b_{1}$ is bounded by 4 . 
For symplectic 4-manifolds with Kodaira dimension 1 or 2 we cannot expect to have a classification: Gompf [6] showed that any finitely presented group is the fundamental group of a symplectic 4-manifold either of Kodaira dimension 1 or of Kodaira dimension 2. Instead one is interested in further illustrating the diversity of simply connected minimal symplectic 4-manifolds ([1], 6], [5], [13]). The problem is to realize all pairs $(\chi(M), \sigma(M))$ of a simply connected minimal symplectic 4 -manifold $M$ subject to the Noether condition $2 \chi+3 \sigma \equiv \sigma(\bmod 8)$ (symplectic manifolds admit almost complex structures) and the conjectured inequality $\chi \geq 3 \sigma$ (the symplectic Bogomolov-MiyaokaYau inequality).

For simply connected minimal symplectic 4-manifolds with Kodaira dimension 1 this has a simple positive answer. Such a manifold has $b_{1}=0$ and $K^{2}=0$, so

$$
2 \chi+3 \sigma=4\left(1-b_{1}+b_{+}\right)+\sigma=0 .
$$

Therefore $\sigma$ is nonpositive and $\chi$ is determined by $\sigma$. And since $M$ is almost complex, $b^{+}-b_{1}$ is odd, and hence $\sigma(M)$ is divisible by 8 .

The Dolgachev surfaces and Elliptic surfaces $E(n)$ with $n \geq 2$ are simply connected minimal symplectic 4-manifolds with Kodaira dimension 1 with signature -8 and $-8 n$ respectively. Hence we have the well-known fact:

Proposition 1 Every negative integer divisible by 8 is the signature of a simply connected minimal symplectic 4-manifold with Kodaira dimension 1.

In this paper we investigate the geography question of minimal symplectic 4manifolds of Kodaira dimension 1 by taking into account the first Betti number $b_{1}$ and the cup product structure on $H^{1}$ (a question perhaps mostly of interest to 4 -manifold topologists). By Equation (11), the pair $(\chi, \sigma)$ is equivalent to $\left(\sigma, b_{1}\right)$ when the Kodaira dimension is 1 . We will use the latter pair of numbers in what follows to make definitions and statements more transparent. The third parameter is determined by the symplectic form and can be formulated in terms of a Kähler-like condition called Lefschetz type.

Definition 2 Symplectic 4-manifolds $(M, \omega)$ are said to be of Lefschetz type if $[\omega] \in H^{2}(M ; \mathbb{R})$ satisfies the conclusion of the Hard Lefschetz Theorem, namely, that $\cup[\omega]: H^{1}(M ; \mathbb{R}) \rightarrow H^{3}(M ; \mathbb{R})$ is an isomorphism.

Based upon that definition one gets: 
Definition 3 The degeneracy $d(M, \omega)$ is the rank of the kernel of the map

$$
\cup[\omega]: H^{1}(M ; \mathbb{R}) \rightarrow H^{3}(M ; \mathbb{R}) .
$$

The first example of a symplectic 4-manifold with nonzero degeneracy occurs when $b_{1}=2$ and $b_{+}=1$. A symplectic 4 -manifold with $b_{+}=1$ and $\kappa=1$ must satisfy $b_{1}=0$ or $b_{1}=2$ using the fact that $2 \chi+3 \sigma=0$. Examples of $b_{1}=2$ 4 -manifolds of Lefschetz type are easy to construct and have been known for some time, but examples of manifolds not of Lefschetz type remained unknown until Baldridge [1. These $b_{+}=1$ manifolds with nonzero degeneracy are the starting point for the examples described in this paper.

Definition 4 Any triple $(a, b, c) \in \mathbb{Z}^{3}$ is called an admissible triple (or Lefschetz admissible) if $a=8 k$ where $k$ is a non-positive integer, $0 \leq c \leq b, b-c$ even, and $b \geq \max \{0,2+a / 4\}$.

In the above definition, $a$ corresponds to the signature of a symplectic 4manifold, $b$ is the first Betti number, and $c$ is the degeneracy. This definition covers all triples except possible counterexamples to the conjectured BMY inequality. The next lemma explains why $b-c$ should be even.

Lemma 5 Let $(M, \omega)$ be a closed symplectic 4-manifold. The skew-symmetric bilinear form $Q_{M}: H^{1}(M ; \mathbb{R}) \times H^{1}(M ; \mathbb{R}) \rightarrow \mathbb{R}$ defined by

$$
Q_{M}(a, b)=\int_{M} a \cup b \cup[\omega]
$$

has rank $b_{1}(M)-d(M, \omega)$. Furthermore, rank $Q_{M}$ is even.

Proof Pick a compatible metric $g$ and set

$$
K=\left\{\alpha \in H^{1}(M ; \mathbb{R}) \mid \alpha \cup[\omega]=0 \in H^{3}(M ; \mathbb{R})\right\} .
$$

$K$ is a closed subspace of $H^{1}(M ; \mathbb{R})$ with rank $d(M, \omega)$. Let $W$ be the orthogonal complement of $K$ in $H^{1}(M ; \mathbb{R})$ with respect to the $L^{2}$-norm on $H^{1}(M ; \mathbb{R})$.

We claim that $Q_{M}$ is nondegenerate on $W$. To see this, suppose $a \in W$ satisfies $Q_{M}(a, b)=0$ for all $b \in W$. If the class $a \cup[\omega]$ is not zero, then by Poincaré duality there exists a 1 -cocycle $\gamma$ such that $Q_{M}(\gamma, a)=\langle\gamma \cup a \cup[\omega],[M]\rangle \neq 0$. Therefore $a \cup[\omega]=0$ and $a \in K$ which implies $a=0$.

Now by the same proof that symplectic forms are locally isomorphic to the standard symplectic form on $\left(\mathbb{R}^{2 n}, \omega_{0}\right)$, rank $Q_{M}$ is even dimensional. 
We can now state the main theorem of this paper:

Theorem 6 For any admissible triple $(a, b, c)$ there exists a minimal symplectic 4-manifold $(M, \omega)$ of Kodaira dimension $\kappa(M)=1$ with

$$
(a, b, c)=\left(\sigma(M), b_{1}(M), d(M, \omega)\right),
$$

where $\sigma(M)$ is the signature and $b_{1}(M)$ is the first Betti number.

\section{An important lemma}

In Section 4 we will produce examples of symplectic 4-manifolds for admissible triples $(0, b, c)$. We will then use these manifolds to fill out triples where $a<0$ in Section 5. To build these manifolds we need a general theorem that was first reported in [4].

Theorem 7 Let $\Sigma$ be a closed, oriented, connected surface of genus $g$ with an orientation preserving diffeomorphism $\varphi: \Sigma \rightarrow \Sigma$. Let $Y$ be the mapping torus with respect to $\Sigma$ and $\varphi$ and $p: Y \rightarrow S^{1}$. Let $\mathfrak{e} \in H^{2}(Y ; \mathbb{Z})$ be any class such that $\left.\mathfrak{e}\right|_{\Sigma}=0$ and let $M \rightarrow Y$ be the $S^{1}$-bundle over $Y$ with Euler class $\mathfrak{e}$. Then $M$ is a smooth oriented closed symplectic 4-manifold.

The advantage of the construction below over the one in 4 is that we can derive the cohomology ring explicitly for the examples we need.

Proof We begin by constructing a basis for the cohomology $H^{1}(Y ; \mathbb{Z})$ from smooth integral 1-forms. Let $\theta \in \Omega^{1}(Y)$ be the pullback of the volume of $S^{1}$. This is a closed, integral, nonzero $1-$ form.

Consider the map $\varphi^{*}-1$ on $H^{1}(\Sigma ; \mathbb{Z})$ and let $k=\operatorname{rank} \operatorname{ker}\left(\varphi^{*}-1\right)$. The Wang exact sequence implies that the rank of $H^{1}(Y)$ is $k+1$ :

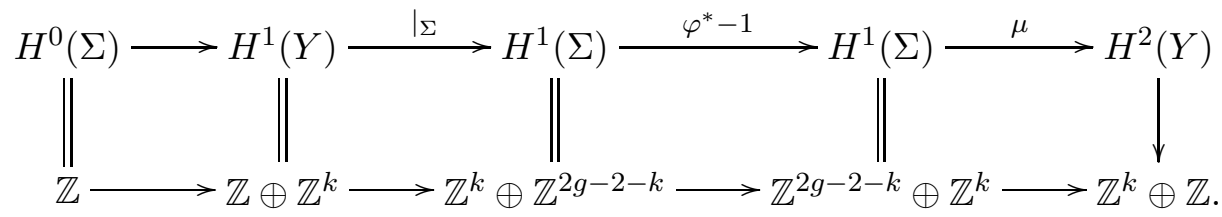

Write down a basis for $H^{1}(Y ; \mathbb{Z})$ as follows. Let $\left\langle\gamma_{1}, \cdots, \gamma_{k}\right\rangle$ be a basis of closed integral 1 -forms on $\Sigma$ for the subspace of $H^{1}(\Sigma ; \mathbb{Z})$ which is preserved by $\varphi^{*}$. Extend this to a basis of $H^{1}(\Sigma ; \mathbb{Z})$ by closed 1 -forms:

$$
\left\langle\left[\gamma_{1}\right], \ldots,\left[\gamma_{k}\right],\left[\epsilon_{k+1}\right], \ldots,\left[\epsilon_{2 g}\right]\right\rangle \text {. }
$$


Because $\left[\gamma_{i}\right]$ is invariant under $\varphi^{*}$ there is a function $f_{i} \in \Omega^{0}(\Sigma)$ such that $\varphi^{*}\left(\gamma_{i}\right)=\gamma_{i}+d f_{i}$ point-wise. To construct a closed 1-form on $Y$ which is non-trivial in cohomology, choose a smooth function $\rho:[0,1] \rightarrow[0,1]$ which is identically 0 near 0 and identically 1 near 1 and extend $\gamma_{i}$ to $\Sigma \times[0,1]$ by defining

$$
\bar{\gamma}_{i}(x, t)=\rho(t) \gamma_{i}(x)+(1-\rho(t))\left(\varphi^{*}\left(\gamma_{i}(x)\right)\right)-\left(\frac{d}{d t} \rho(t)\right) f_{i}(x) d t .
$$

Since $\bar{\gamma}_{i}$ can be identified on the boundary of $\Sigma \times[0,1]$ using $\varphi^{*}$, and

$$
\left.\frac{d^{n}}{d t^{n}} \varphi^{*}\left(\bar{\gamma}_{i}\right)\right|_{\Sigma \times\{0\}}=\left.\frac{d^{n}}{d t^{n}} \bar{\gamma}_{i}\right|_{\Sigma \times\{1\}}
$$

for all whole numbers $n, \bar{\gamma}_{i}$ is a closed smooth 1 -form on $Y$. Hence,

$$
H^{1}(Y ; \mathbb{R})=\left\langle[\theta],\left[\bar{\gamma}_{1}\right], \ldots,\left[\bar{\gamma}_{k}\right]\right\rangle .
$$

Next we construct a basis of $H^{2}(Y)$. First, there is a smooth closed integral 2-form $\Omega_{\Sigma} \in \Omega^{2}(Y)$ which restricts to the volume form of $\Sigma$ on each fiber of $p: Y \rightarrow S^{1}$. Writing down a basis which spans $\operatorname{Im}\left(\mu: H^{1}(\Sigma) \rightarrow H^{2}(Y)\right)$ in the Wang sequence is crucial to understanding what symplectic 4 -manifolds can be built from $Y$. Note that

$$
\left.\varphi_{*}\left(P D\left(\gamma_{i}\right)\right)=\varphi_{*}\left(\gamma_{i} \cap[\Sigma]\right)=\varphi_{*}\left(\varphi^{*}\left(\gamma_{i}\right)\right) \cap[\Sigma]\right)=\gamma_{i} \cap \varphi_{*}[\Sigma]=P D\left(\gamma_{i}\right),
$$

where $P D$ is the Poincaré dual. Hence $P D\left(\gamma_{i}\right)$ is $\varphi$-invariant in homology and $\left\langle t, P D\left(\gamma_{1}\right), \ldots, P D\left(\gamma_{k}\right)\right\rangle$ is a basis for $H_{1}(Y)$ where $t$ is the loop $p t \times[0,1]$ in $\Sigma \times[0,1]$ for some fixed point of $\varphi$.

Let $\xi_{i}$ be a 1 -form which is the Hom-dual of $P D\left(\gamma_{i}\right)$. Extend the linearly independent set $\left\langle\left[\xi_{1}\right], \ldots,\left[\xi_{k}\right]\right\rangle$ to a basis of $H^{1}(\Sigma)$ given by

$$
\left\langle\left[\xi_{1}\right], \ldots,\left[\xi_{k}\right],\left[\zeta_{k+1}\right], \ldots\left[\zeta_{2 g}\right]\right\rangle \text {. }
$$

The classes $\left[\xi_{i}\right]$ are not necessarily $\varphi^{*}$-invariant. Nevertheless, since

$$
\left\langle\varphi^{*}\left(\xi_{i}\right), P D\left(\gamma_{j}\right)\right\rangle=\left\langle\xi_{i}, \varphi_{*}\left(P D\left(\gamma_{j}\right)\right)\right\rangle=\left\langle\xi_{i}, P D\left(\gamma_{j}\right)\right\rangle=\delta_{i j},
$$

there exists functions $g_{i} \in \Omega^{0}(\Sigma)$ and integers $c_{j}$ such that

$$
\varphi^{*}\left(\xi_{i}\right)=\xi_{i}+\sum_{j=k+1}^{2 g} c_{j} \zeta_{j}+d g_{i}
$$

point-wise. Using $\xi_{i}$ and $g_{i}$, construct a smooth 1-form on $Y$ by specifying

$$
\bar{\xi}_{i}(x, t)=\rho(t) \xi_{i}(x)+(1-\rho(t))\left(\varphi^{*}\left(\xi_{i}(x)\right)\right)-\left(\frac{d}{d t} \rho(t)\right) g_{i}(x) d t .
$$


This 1 -form is not necessarily closed, in fact,

$$
d \bar{\xi}_{i}=\left(\sum c_{j} \zeta_{j}\right) \wedge\left(\frac{d}{d t} \rho\right) \theta
$$

However $\bar{\xi}_{i} \wedge \theta$ is a smooth closed integral 2-form and

$$
\left\langle\left[\Omega_{\Sigma}\right],\left[\bar{\xi}_{1} \wedge \theta\right], \ldots,\left[\bar{\xi}_{k} \wedge \theta\right]\right\rangle
$$

is a basis for $H^{2}(Y ; \mathbb{Z})$.

We can now complete the proof of Theorem $\mathbf{7}$. If $\mathfrak{e} \in H^{2}(Y ; \mathbb{Z})$ such that $\left.\mathfrak{e}\right|_{\Sigma}=0$, then

$$
\mathfrak{e}=\sum_{i=1}^{k} p_{i}\left[\bar{\xi}_{i} \wedge \theta\right]
$$

where $p_{i} \in \mathbb{Z}$. Let $M$ be the $S^{1}$-bundle over $Y$ with Euler class $\mathfrak{e}$ and connection 1-form $\eta$ given by $d \eta=\sum_{i=1}^{k} p_{i} \pi^{*}\left(\bar{\xi}_{i} \wedge \theta\right)$ point-wise. Then

$$
\omega=\pi^{*}(\Omega)+\pi^{*}(\theta) \wedge \eta
$$

is an everywhere nondegenerate 2 -form on $M$ such that

$$
d \omega=-\pi^{*}(\theta) \wedge d \eta=-\pi^{*}(\theta) \wedge \sum_{i=1}^{k} p_{i} \pi^{*}\left(\bar{\xi}_{i} \wedge \theta\right)=0 .
$$

This ends the proof of the theorem.

McDuff and Salamon [12] raised the question whether there is a free symplectic circle action on symplectic 4-manifold with contractible orbits. They pointed out that null-homologous orbits exists on certain $T^{2}$-bundles over $T^{2}$. Here we show that there cannot be any contractible orbits.

Proposition 8 Suppose $S^{1}$ acts freely (fixed point free) on $(M, \omega)$ preserving $\omega$. Then the orbits are essential in $\pi_{1}(M) 1$

Proof Let $Y=M / S^{1}$. Then it is known that $Y$ fibers over $S^{1}$. When the fiber is of genus at least one, $Y$ is a $K(\pi, 1)$ space from the homotopy exact sequence associated to this fibration, in particular it has trivial $\pi_{2}$. Consider the homotopy exact sequence associated to the fibration $S^{1} \longrightarrow M \longrightarrow Y$,

$$
\cdots \longrightarrow \pi_{2}(Y) \longrightarrow \pi_{1}\left(S^{1}\right) \longrightarrow \pi_{1}(M) \longrightarrow \cdots .
$$

The orbits are contractible implies that $\pi_{2}(Y)$ surjects onto $\pi_{1}\left(S^{1}\right)=\mathbf{Z}$. This is a contradiction.

${ }^{1}$ Added in proof: We have learned that this is a special case of a result of Kotschick 7. Theorem 1], where the action is not assumed to preserve the symplectic structure in dimension 4. Moreover examples of symplectic free actions with contractible orbits in every dimension at least 6 are constructed in [7, Theorem 2]. 


\section{Bundle manifolds}

In this section we describe symplectic 4-manifolds which are special $S^{1}$-bundles over a base which is itself a surface bundle over $S^{1}$, which we will call bundle manifolds. They are uniquely specified by four whole numbers: three weights $g, d, k$ where $0 \leq d \leq k \leq g$ and a number $e=0,1,2$ based upon the Euler class of the $S^{1}$-bundle. We denote these manifolds by $B(d, k, g ; e)$.

Construct a bundle manifold as follows. Let $\Sigma$ be a surface of genus $g>0$. Let $\left\langle a_{1}, b_{1}, \ldots a_{g}, b_{g}\right\rangle$ be smooth loops which represent the usual symplectic basis of $H_{1}(\Sigma)$ such that $a_{i} \cdot b_{j}=\delta_{i j}$ and zero otherwise.

Similarly, let $\left\langle\alpha_{1}, \beta_{1}, \ldots \alpha_{g}, \beta_{g}\right\rangle$ be closed integral 1-forms which represent a dual basis with respect to the $a_{i}$ 's and $b_{i}$ 's:

$$
\alpha_{i}\left(a_{j}\right)=\delta_{i j}, \quad \beta_{i}\left(b_{j}\right)=\delta_{i j}, \quad \text { and zero otherwise. }
$$

Consider the diffeomorphism given by the following sequence of Dehn twists acting on the left,

$$
\varphi=\left(T_{b_{g}} T_{a_{g}}^{-1}\right) \cdots\left(T_{b_{k+1}} T_{a_{k+1}}^{-1}\right) \cdot T_{a_{d}} \cdots T_{a_{1}} .
$$

This diffeomorphism has the following properties:

- For each $1 \leq i \leq d$ when $d \neq 0$, the subspace spanned by $\left\langle\alpha_{i}, \beta_{i}\right\rangle$ has a 1 -dimensional subspace preserved by $\varphi^{*}$,

$$
\varphi^{*} \alpha_{i}=\alpha_{i}+\beta_{i} \quad \text { and } \quad \varphi^{*} \beta_{i}=\beta_{i}
$$

as cohomology classes.

- For each $d<i \leq k$ when $d \neq k$, the subspace spanned by $\left\langle\alpha_{i}, \beta_{i}\right\rangle$ is preserved by $\varphi^{*}: H^{1}(\Sigma ; \mathbb{Z}) \rightarrow H^{1}(\Sigma ; \mathbb{Z})$.

- The subspace spanned by $\left\langle\alpha_{k+1}, \beta_{k+1}, \ldots \alpha_{g}, \beta_{g}\right\rangle$ contains no subspace which is preserved by $\varphi^{*}$.

The mapping torus

$$
Y=(\Sigma \times[0,1]) /((x, 1) \sim(\varphi(x), 0))
$$

is a smooth, closed, 3-dimensional manifold which, by the proof of Theorem 7 has the following basis in cohomology:

$$
\begin{aligned}
H^{1}(Y)= & \left\langle\theta, \quad \bar{\beta}_{1}, \ldots, \bar{\beta}_{d}, \quad \bar{\alpha}_{d+1}, \bar{\beta}_{d+1}, \ldots, \bar{\alpha}_{k}, \bar{\beta}_{k}\right\rangle \\
H^{2}(Y)= & \left\langle\Omega, \quad \bar{\alpha}_{1} \wedge \theta, \ldots, \bar{\alpha}_{d} \wedge \theta,\right. \\
& \left.\bar{\alpha}_{d+1} \wedge \theta, \bar{\beta}_{d+1} \wedge \theta, \ldots, \bar{\alpha}_{k} \wedge \theta, \bar{\beta}_{k} \wedge \theta\right\rangle .
\end{aligned}
$$


Also by Theorem [7 any $S^{1}$-bundle over $Y$ is symplectic as long as the Euler class $\mathfrak{e} \in H^{2}(Y ; \mathbb{Z})$ is zero when restricted to a fiber $\Sigma$. Therefore we can specify a symplectic 4 -manifold $B(d, k, g ; e)$ using the diffeomorphism $\varphi$ by choosing four whole numbers $g, d, k, e$ such that $0 \leq d \leq k \leq g$ and

$$
e=\left\{\begin{array}{l}
0 \mathfrak{e}=0, \\
1 \mathfrak{e}=\left[\bar{\alpha}_{i} \wedge \theta\right] \text { for some } 0<i \leq d, d \neq 0 \\
2 \mathfrak{e} \in \operatorname{span}\left\langle\left[\bar{\alpha}_{d+1} \wedge \theta\right],\left[\bar{\beta}_{d+1} \wedge \theta\right], \ldots,\left[\bar{\beta}_{k} \wedge \theta\right]\right\rangle, \mathfrak{e} \text { primitive, and } d \neq k .
\end{array}\right.
$$

Remark 9 The manifolds described in [1] are just the bundle manifolds given by $B(1,1, g ; 1)$ for all genera $g>1$.

The bundle manifold $B(d, k, g ; e)$ does not depend on the choice of an Euler class $\mathfrak{e} \in H^{2}(Y)$; two bundle manifolds with different Euler classes but with the same data $g, d, k, e$ are diffeomorphic.

The following lemmas will be helpful in the next section.

Lemma 10 The Kodaira dimension of a bundle manifold $B(d, k, g ; e)$ is $\kappa=0$ if $g=1$ and $\kappa=1$ if $g>1$.

Proof This theorem follows using similar arguments as in [1].

Lemma 11 The signature of $B(d, k, g ; e)$ is zero.

Proof This is an easy computation given the existence of a free circle action on bundle manifolds.

Lemma 12 The first Betti number of the bundle manifold $B(d, k, g ; e)$ is:

$$
b_{1}= \begin{cases}2 k-d+2 & e=0 \\ 2 k-d+1 & e \neq 0\end{cases}
$$

Proof The Gysin Sequence,

$$
0 \longrightarrow H^{1}(Y) \stackrel{\pi^{*}}{\longrightarrow} H^{1}(B(d, k, g ; e)) \longrightarrow H^{0}(Y) \stackrel{\cup \mathfrak{e}}{\longrightarrow} H^{2}(Y),
$$

implies that

$$
H^{1}(B(d, k, g ; e), \mathbb{Z}) \cong \begin{cases}H^{1}(Y, \mathbb{Z}) \oplus \mathbb{Z}, & e=0 \\ H^{1}(Y ; \mathbb{Z}), & e \neq 0\end{cases}
$$

The first Betti number can then be calculated using the basis constructed in Equation (3). 
Lemma 13 The degeneracy $d((B(d, k, g ; e), \omega)$ is

$$
d(B(d, k, g ; e), \omega))=\left\{\begin{array}{cc}
d & e=0 \\
d+1 & e \neq 0
\end{array}\right.
$$

Proof We prove the case when $e=0$, i.e., $B(d, k, g ; 0)=Y \times S^{1}$, noting that the case when $e \neq 0$ is similar. Let $t$ be a section of the fibration $Y \rightarrow S^{1}$ such that $\langle\theta,[t]\rangle=1$. A basis for $H_{3}\left(Y \times S^{1} ; \mathbb{Z}\right)$ can be described as follows. By construction, $\varphi_{*}\left(a_{i}\right)=a_{i}$ for $1 \leq i \leq d$. While $a_{i}$ is null-homologous due to the relation created by $\varphi_{*}\left(b_{i}\right)=a_{i}+b_{i}$, the 2-cycle $a_{i} \times t$ is not, and the space generated by $\left\{a_{i} \times t \times S^{1} \mid 1 \leq i \leq d\right\}$ forms a linearly independent subspace of $H_{3}\left(Y \times S^{1} ; \mathbb{Z}\right)$. For $d<i \leq k, \varphi_{*}$ is identity on $a_{i}$ 's and $b_{i}$ 's, and so the space generated by $\left\{a_{i} \times t \times S^{1}, b_{i} \times t \times S^{1} \mid d<i \leq k\right\}$ is also a linearly independent subspace. Altogether, a basis for homology 3-cycles of $Y \times S^{1}$ is

$$
\begin{aligned}
H_{3}\left(Y \times S^{1} ; \mathbb{Z}\right)= & \left\langle[Y], \Sigma \times S^{1}, a_{1} \times t \times S^{1}, \ldots, a_{d} \times t \times S^{1},\right. \\
& \left.a_{d+1} \times t \times S^{1}, b_{d+1} \times t \times S^{1}, \ldots, b_{k} \times t \times S^{1}\right\rangle .
\end{aligned}
$$

Using the basis described in Equation (3) we can determine the kernel of $\cup \omega$ : $H^{1}\left(Y \times S^{1} ; \mathbb{R}\right) \rightarrow H^{3}\left(Y \times S^{1} ; \mathbb{R}\right)$. Clearly $\theta \wedge \omega=\pi^{*}(\theta \wedge \Omega)$ evaluates nonzero on $[Y]$, so $\theta$ is not in the kernel. Similarly, $\eta$ (the connection 1-form for the $S^{1}$ factor of $\left.Y \times S^{1}\right)$ is not in the kernel because $\eta \wedge \omega=\eta \wedge \pi^{*} \Omega$ evaluates nonzero on $\Sigma \times S^{1}$. Observe that $\left\langle\bar{\alpha}_{i} \wedge \omega, a_{i} \times t \times S^{1}\right\rangle \neq 0$ and $\left\langle\bar{\beta}_{i} \wedge \omega, b_{i} \times t \times S^{1}\right\rangle \neq 0$ for $d<i \leq k$. Finally, $\bar{\beta}_{i} \wedge \omega$ 's for $1 \leq i \leq d$ evaluates zero on all 3 -cycles except possibly $a_{i} \times t \times S^{1}$. However $\left\langle\bar{\beta}_{i} \wedge \omega, a_{i} \times t \times S^{1}\right\rangle= \pm\left\langle\beta_{i}, a_{i}\right\rangle=0$. Therefore the subspace generated by $\bar{\beta}_{i}$ 's for $1 \leq i \leq d$ is the kernel of $\cup \omega$ implying that $d(B(d, k, g ; 0)=d$.

\section{Examples of admissible triples with $a=0$}

We first prove Theorem 6 for admissible triples $(a, b, c)$ when $a=0$ (i.e. where the signature of the manifold is zero). In the next section we use these manifolds to construct new manifolds for admissible triples when $a<0$.

The case where $a=0$ and $b$ is even Fix $b=2 l$ for some whole number $l>$ 0 . We are looking for symplectic $4-$ manifolds $(M, \omega)$ with Kodaira dimension 1 and $\sigma(M)=0, b_{1}(M)=2 l$, and even $d(M, \omega)$ where $0 \leq d(M, \omega) \leq 2 l$. When we restrict to bundle manifolds with $e=0$, we get by Lemma 12 that

$$
d=2(k-l+1) \text {. }
$$


Since $0 \leq d \leq k$, the possible bundle manifolds occur when $k=l-1, \cdots, 2 l-2$. This, together with Lemma 13] implies that the infinite collection of symplectic 4 -manifolds

$\left\{B_{0}(i):=B(2 i, l-1+i, g ; 0) \quad \mid i=0,1, \ldots, l-1\right.$ and $\left.g \geq \max (l-1+i, 2)\right\}$ satisfy

$$
\begin{aligned}
b_{1}\left(B_{0}(i)\right) & =2 l=b \text { and } \\
d\left(B_{0}(i), \omega\right) & =2 i .
\end{aligned}
$$

This leaves only the case of an admissible triple $(0, b, c)$ where $c=b$. For that we investigate the case when $e=1$. Then by Lemma[12, bundle manifolds with $e=1$ exist, and satisfy

$$
d=2(k-l)+1
$$

when $1 \leq d \leq k$. Hence possible values of $k=l, l+1, \ldots, 2 l-1$ and the infinite set of bundle manifolds

$$
\left\{B_{1}(i):=B(2 i+1, l+i, g ; 1) \mid i=0,1, \ldots, l-1 \text { and } g \geq \max (l+i, 2)\right\}
$$

satisfy

$$
\begin{aligned}
b_{1}\left(B_{1}(i)\right) & =2(l+i)-(2 i+1)+1=b \text { and } \\
d\left(B_{1}(i), \omega\right) & =(2 i+1)+1=2(i+1)
\end{aligned}
$$

by Lemma 12 and Lemma 13 Therefore the manifold $B_{1}(l-1)$ is an example of an admissible triple $(0, b, b)$. This ends the case when $b$ is even.

The case when $a=0$ and $b$ is odd Fix $b=2 l+1$ where $l>0$. We restrict to bundle manifolds where $e \neq 0$. Lemma 12 implies that $d$ is a function of $k$,

$$
d=2(k-l),
$$

where $k$ can equal $l$ only if $e=2, k=2 l$ only if $e=1$, and $k=l+1, \ldots, 2 l-1$ for $e=1$ or 2 . The infinite set of bundle manifolds

$$
\begin{array}{lll}
B_{1}(i):=B(2 i, l+i, g ; 1) & i=1, \ldots, l \text { and } g \geq \max (l+i, 2), \\
B_{2}(i):=B(2 i, l+i, g ; 2) & i=0,1, \ldots, l-1 \text { and } g \geq \max (l+i, 2),
\end{array}
$$

satisfies

$$
\begin{aligned}
b_{1}\left(B_{e}(i)\right) & =2(l+i)-2 i+1=b \text { and } \\
d\left(B_{e}(i), \omega\right) & =2 i+1
\end{aligned}
$$

for $e=1$ or 2 by Lemma 12 and Lemma 13. This completes the proof of Theorem [6 for the case when $a=0$.

Remark 14 We could have generated examples for $b$ odd by looking at bundle manifolds with $e=0$. In that case there are examples for $b-c$ even and $b \neq 1$ except for the admissible triple $(0, b, c)$ where $b=c$. 


\section{Proof of Theorem 6}

To fill out all admissible triples $(a, b, c)$ for $a<0$ and divisible by 8 , we fibersum $E(n)$ with bundle manifolds. This turns out to be much easier than the $a=0$ case since we need only work with $B(d, k, g ; e)$ where $e=0$.

Inside $B(d, k, g ; 0)$ we can find a symplectic torus $T$ with $T \cdot T=0$, constructed as follows. Since $B(d, k, g ; 0)=Y \times S^{1}$, recall the definition of $Y$ and let $t$ be a section of $p: Y \rightarrow S^{1}$. Then $T=\pi^{-1}(t)$ is a nontrivial torus in $B(d, k, g ; 0)$ which clearly has $T \cdot T=0$. Letting $s$ be the loop generated by the $S^{1}$ factor of $Y \times S^{1}$, we can write $T=t \times s \in H_{2}(B(d, k, g ; 0) ; \mathbb{Z})$. Since $\omega \mid T \neq 0$ point-wise, $T$ is a symplectic submanifold.

Next let $T^{\prime}$ be a generic torus fiber of $E(n)$ in the neighborhood of a cusp fiber. Consider the fibersum

$$
E(n, d, k, g)=E(n) \#_{T^{\prime}=T} B(d, k, g ; 0)
$$

where $n \geq 2$. Since we are fibering along two symplectic surfaces, $E(n, d, k, g)$ is symplectic with symplectic form $\tilde{\omega}$. It was proved in [9] that fiber-sums of minimal manifolds are minimal. Therefore all the manifolds above are minimal.

Clearly the two loops $t$ and $s$ become null-homologous in $H_{1}(E(n, d, k, g) ; \mathbb{Z})$ after identifying along the boundary, making $b_{1}(E(n, d, k, g)=2 k-d$ by Lemma 12. Also by the Novikov Signature Theorem,

$$
\sigma(E(n, d, k, g))=\sigma(E(n))+\sigma(B(d, k, g ; 0))=-8 n .
$$

We need to check that $\kappa(E(n, d, k, g))=1$. Since the Poincaré dual of the canonical bundle $P D\left(K_{E(n)}\right)=(n-2) T^{\prime}$ and the Poincaré dual of the canonical bundle $P D\left(K_{B(d, k, g, 0)}\right)=(2 g-2) T$, the fiber sum $E(n, d, k, g)$ has canonical class $P D(K)=(n-2+2 g) T$. Therefore $K^{2}=0$ and $K \cdot[\tilde{\omega}]=n-2+2 g>0$ when $n>1$ and $g>0$.

To finish the proof of Theorem [6] we need a symplectic 4-manifold with $\kappa=1$ for the admissible triple $(a, b, c)$, where $a<0$. Since $0 \leq c \leq b$ with $b-c$ even, set $k=(b+c) / 2$ and note that $0 \leq c \leq k$ by the fact that $0 \leq 2 c \leq b+c$. Then $E(-a / 8, c, k, g)$ is the desired manifold for $g \geq k$, after observing the next lemma.

Lemma 15 The degeneracy of $E(n, d, k, g)$ is $d$.

Proof It is straight forward to compute the ring structure of $B(d, k, g, 0)$ given that it is a product of a three manifold with $S^{1}$ (See Lemma 13 for an example of such calculations). 
For symplectic manifolds with signature -8 , we fibersum with Dolgachev surfaces $E(1)_{p, q}$ instead of $E(1)$ to get a minimal symplectic 4-manifold.

\section{$6 \quad$ Null admissible triples}

We do not know how much the degeneracy of a symplectic manifold $(M, \omega)$ depends on the symplectic form $\omega$. It may be true that $d(M, \omega) \neq d\left(M, \omega^{\prime}\right)$ for two different symplectic forms $\omega$ and $\omega^{\prime}$ on the same manifold. One could look at another invariant, called the nullity of $M$, which only depends on the ring structure of $M$ and not on the particular symplectic form chosen.

Definition 16 For any $\alpha \in H^{1}(M ; \mathbf{R})$, and $i=1,2$, consider the map

$$
i_{\alpha}=\cup \alpha: H^{i}(M ; \mathbf{R}) \longrightarrow H^{i+1}(M ; \mathbf{R}) .
$$

The dimension of the linear space $\left\{\alpha \mid i_{\alpha}=0\right\}$ is called the $i-n u l l i t y$ of $M$, denoted $n_{i}(M)$.

Lemma $17 n_{1}(M)=n_{2}(M)$.

Proof If $1_{\alpha}=0$, then we claim that $2_{\alpha}=0$. Otherwise there is a $\gamma \in$ $H^{2}(M ; \mathbf{R})$ such that $\alpha \cup \gamma$ is nonzero in $H^{3}(M ; \mathbf{R})$. By the Poincaré Duality, there is a class $\beta \in H^{1}(M ; \mathbf{R})$ such that $(\alpha \cup \gamma) \cup \beta \neq 0$. This implies that $1_{\alpha}(\beta)=\alpha \cup \beta \neq 0$, which is a contradiction. Similarly we can prove that $2_{\alpha}=0$ implies that $1_{\alpha}=0$.

Thus we can speak simply of nullity of $M, n(M)$.

It follows from Lemma 17 that the nullity is a lower bound for the degeneracy of $M$, i.e., $d(M, \omega) \geq n(M)$. From Hodge theory we get that Kähler surfaces are of Lefschetz type, and hence have nullity zero.

One can also talk about triples $(a, b, c)$ where $c$ is the nullity of a Kodaira dimension 1 symplectic manifold (or for a symplectic manifold in general).

Definition 18 Any triple $(a, b, c) \in \mathbb{Z}^{3}$ is called null admissible if $a=8 k$ where $k$ is a non-positive number, $0 \leq c \leq b, c \neq b-1$, and $b \geq \max \{0,2+a / 4\}$.

Observe that we require that $c \neq b-1$, for if the nullity of a manifold $M$ was $b_{1}(M)-1$, there would be an element in $H^{1}(M ; \mathbb{Z})$ whose cup product square would not be zero. Note that we no longer have a reason to require that $b-c$ be even. In fact, the next lemma shows that $b-c$ may be even or odd. 
Lemma 19 The nullity $n(B(d, k, g ; e))$ is

$$
n(B(d, k, g ; e))=\left\{\begin{array}{cl}
0 & e=0 \\
d & e \neq 0, d \neq k \\
d+1 & e \neq 0, d=k
\end{array}\right.
$$

Proof Compute the ring structure for cup products on $H^{1}(B(d, k, g ; e) ; \mathbb{R})$ using the explicit basis given in Equation (3) and in the proof of Lemma 13.

We can use Lemma 19 to find null admissible triples for Kodaira dimension 1. For example, when $a=0$ and $b=2$ (recall that $b$ can not equal 1 ), the lemma shows that $B(0,0, g ; 0)$ has nullity zero and that $B(1,1, g ; 1)$ has nullity 2 ; the triple $(0,2,1)$ is not a null admissible triple.

For the case when $a=0$ and $b=3$, we can recognize the null admissible triples $(0,3,0)$ and $(0,3,3)$ using bundle manifolds $B(1,1, g ; 0)$ and $B(2,2, g ; 1)$ respectively. Lemma 19 does not give an example for the null admissible triple $(0,3,1)$. The ring multiplication for such a manifold would have to look like

\begin{tabular}{c|ccc} 
& $a$ & $b$ & $c$ \\
\hline$a$ & 0 & $a \cup b$ & 0 \\
$b$ & $b \cup a$ & 0 & 0 \\
$c$ & 0 & 0 & 0
\end{tabular}

for a basis $\langle a, b, c\rangle$ of $H^{1}(M ; \mathbb{Z})$ where $a \cup b \neq 0$. We end this report with the following question.

Question 20 Does there exist a symplectic 4-manifold $(M, \omega)$ with $\sigma(M)=$ $0, b_{1}(M)=3$, and nullity $n(M)=1$ ?

Acknowledgements The first author is partially supported by NSF grant DMS-0406021. The second author is partially supported by NSF and the McKnight fellowship.

\section{References}

[1] S Baldridge, New symplectic 4-manifolds with $b_{+}=1$, preprint.

[2] H Geiges, Symplectic Structures on $T^{2}$-bundles over $T^{2}$, Duke Mathematical Journal 67 (1992), no. 3, 539-555. MathReview 
[3] R Gompf, A Stipsicz, 4-Manifolds and Kirby Calculus, Graduate Studies in Mathematics 20, American Mathematical Society, Providence, Rhode Island, 1999. MathReview

[4] M Fernández, A Gray, J Morgan, Compact symplectic manifolds with free circle actions, and Massey products, Michigan Math. J. 38 (1991), 271-283. MathReview

[5] R Fintushel, R Stern, Knots, links, and 4-manifolds, Invent. Math., 134 (1998), 363-400. MathReview

[6] R Gompf, A new construction of symplectic manifolds, Ann. of Math., 2:142 (1995), 527-595. MathReview

[7] D Kotschick, Free circle actions with contractible orbits on symplectic manifolds, to appear in Math Z.

[8] T J Li, Symplectic 4-manifolds with Kodaira dimension zero, preprint.

[9] T J Li, A Stipsicz, Minimality of certain normal connected sums, Turkish J. Math., 25 (2001), 75-81. MathReview

[10] A Liu, Some new applications of the general wall crossing formula, Math. Res. Letters 3 (1996), 569-585. MathReview

[11] J McCarthy, J Wolfson, Symplectic normal connect sum, Topology 33 (1994), 729-764. MathReview

[12] D McDuff, D Salamon, Introduction to Symplectic topology, Second edition. Oxford Mathematical Monographs. The Clarendon Press, Oxford University Press, New York, 1998. MathReview

[13] A Stipsicz, Simply connected symplectic 4-manifolds with positive signature, Proceedings of 6th Gökova Geometry-Topology Conference, Turkish J. Math. 23 (1999), no. 1, 145-150. MathReview

[14] W Thurston, Some simple examples of symplectic manifolds, Proc. Amer. Math. Soc. 55 (1976), 467-468. MathReview

Department of Mathematics, Louisiana State University

Baton Rouge, LA 70803, USA

and

School of Mathematics, University of Minnesota

Minneapolis, MN 55455, USA

Email: sbaldrid@math.lsu.edu and tjli@math.umn.edu

Received: 22 January 2005 Revised: 30 March 2005 